\title{
Pain and Opioid Use in Chronic Liver Disease: Optimal Treatment Must Address the Mental Health Care Needs of the Patient
}

\author{
Jennifer M. Loftis • Peter Hauser
}

Published online: 20 August 2013

(C) Springer Science+Business Media New York (Outside the USA) 2013

\section{Introduction}

Pain is common and can be challenging to treat among patients with chronic liver disease. Non-steroidal antiinflammatory medications may be underutilized due to their effects on the liver, and opiates may present unique challenges in this population of patients due to concurrent substance use disorder diagnoses and relative lack of efficacy. A better understanding of the factors involved and associated with pain may reveal important insights that promise to improve pain management in this population. Based on studies published to date, routinely screening for and treating co-morbid psychiatric disorders, and, in particular, depressive disorders, would improve the alleviation of pain in people with chronic liver disease.

\section{Pain, Liver Disease, and Substance Use Disorders: Focus on Hepatitis C}

Pain is prevalent in people with chronic hepatitis $\mathrm{C}$ viral (HCV) infection. In a large retrospective study of $\mathrm{HCV}$ -

\footnotetext{
J. M. Loftis

Research \& Development Service, Portland VA Medical Center, 3710 SW U.S. Veterans Hospital Rd., Portland, OR 97239-3098, USA
}

J. M. Loftis

Department of Psychiatry, Oregon Health \& Science University, 3181 SW Sam Jackson Park Rd., Portland, OR 97239-3098, USA

P. Hauser $(\bowtie)$

VISN 22 Network Office, 300 Oceangate, Suite 700,

Long Beach, CA 90802, USA

e-mail: Peter.Hauser2@va.gov infected US Veterans, Whitehead and colleagues [1] reported that $67 \%$ had one or more co-morbid pain-related diagnoses (excluding abdominal pain/stomach discomfort), $56 \%$ had substance use disorder (SUD) diagnoses, $30 \%$ had been prescribed an opioid in the past year, and $46 \%$ had been prescribed an opioid in the past 3 years. Similarly, a second study found that $82.7 \%$ of HCV-infected Veterans reported pain symptoms, which were often chronic, with $65 \%$ of participants reporting pain for 1 year or more [2].

Chronic substance use is associated with high rates of pain [3]. Intravenous drug use is the primary cause of $\mathrm{HCV}$ infection and prior studies suggest 64-90\% of HCV-infected individuals have a history of SUD $[4,5]$. Thus, use of opioid analgesic medications in $\mathrm{HCV}$-infected individuals is of concern to clinicians given the high rates of concurrent SUD in these patients [6, 7]. In the Whitehead study, Veterans with SUD (primarily alcohol use disorders) were significantly more likely to have been diagnosed with a painrelated disorder than those without a SUD [1]. Significant differences were reported between groups for arthropathy, low back pain, and arthritis/rheumatism, but not neuropathy and migraines. Veterans with co-morbid SUD were more likely to have these pain-related diagnoses, suggesting that people with co-morbid SUDs may have a greater sensitivity to pain or a lower pain threshold. Nevertheless, Veterans with HCV and co-morbid SUD were less likely than those without co-morbid SUD to have been prescribed opioids, and were no more likely to fill opioid prescriptions early, suggesting that clinicians are cautious when prescribing opioids to individuals diagnosed with SUDs.

In their recent retrospective study involving chronic liver disease published in this issue, Rogal et al. [8] highlight the prevalence of pain and opioid use associated in a population with chronic liver disease predominantly due to 
$\mathrm{HCV}$ infection. Among diverse factors, they reported that the strongest predictors of pain were emotional distress, mood symptoms, sleep disturbance/fatigue, and advanced liver disease. Rogal et al. note that SUD diagnoses were not prospectively or systematically assessed in their study, with few patients endorsing ongoing substance abuse. Recognizing and understanding the risk and predictive factors associated with pain and opioid use, particularly psychological factors, may provide valuable clues for managing pain and may provide more targeted intervention strategies for patients with chronic liver diseases.

\section{Pain, Liver Disease, and Depression}

Biopsychosocial factors that may be related to the high rates of pain-related disorders among people with liver diseases include psychiatric disorders and in particular depressive disorders. Depression is common among people with $\mathrm{HCV}$ [9], and depression severity has been found to account for the variance in pain severity and pain functioning scores even after controlling for demographic characteristics, opioid prescription status, and disease-related variables [10]. Consistent with these results are other studies that establish a significant relationship between chronic pain and depression $[11,12]$. While the etiology of pain and pain-related diagnoses in chronic liver diseases is, as yet, not clearly elucidated, pro-inflammatory cytokines have been implicated in co-morbid depression and pain, since pro-inflammatory cytokine elevations in $\mathrm{HCV}$-infected individuals correlate with the severity of depressive symptoms [13-15].

A significant proportion of Veterans with $\mathrm{HCV}$ are treated with antidepressants [16]. Rogal et al. reported high rates of antidepressant use in people diagnosed with chronic liver disease. In their study, antidepressant use (excluding tricyclic antidepressants) was more common among those with co-morbid pain than those without comorbid pain (42 vs. $28 \%, P<0.0001$ ), and antidepressants were the most commonly prescribed medication followed by opioids and mood stabilizers [8]. Antidepressants may have indirect anti-inflammatory effects and may partially reverse dysregulation of inflammatory factors [17]. For example, fluoxetine treatment for depression reduces serum interleukin (IL)-6 concentrations in patients [18], and imipramine, clomipramine, venlafaxine, fluoxetine, sertraline, and trazodone reduce plasma pro-inflammatory interferon-gamma concentration relative to anti-inflammatory IL-10 concentration, consistent with an anti-inflammatory effect of many classes of antidepressants [18, 19]. Moreover, non-responders to selective serotonin reuptake inhibitor medications continue to have elevated IL-6 plasma concentrations, suggesting that response to treatment is linked to a reduction of IL-6 [20]. Taken together, these studies suggest a synergistic relationship among $\mathrm{HCV}$, depression, and pain that may be mediated by alterations in immune system function and pro-inflammatory cytokine expression.

\section{Conclusions}

The vast majority of studies that have examined the relationship among pain, liver disease, and psychological factors have been retrospective and cross-sectional in design. Since the studies published to date were correlational, it is not clear whether chronic pain conditions precede depression/emotional distress or vice versa. There are data suggesting that chronic pain is more likely to lead to depression [21], but in patients with chronic liver disease there may be other factors (e.g., alterations in immune system function and cytokine expression) that contribute to both pain and psychiatric symptoms. Prospective, longitudinal studies will be needed to understand the complex relationships among depression, pain, pro-inflammatory cytokines, and SUDs in people with liver disease. As Rogal et al. note, a better understanding of these relationships may reduce pain symptoms with consequent reduced need for opioid use in patients with chronic liver disease [8]. Their study also reinforces the need to screen for and to treat co-morbid depression. More generally, their study suggests that interventions targeted to address the psychiatric co-morbidities that accompany and perpetuate chronic pain would greatly benefit patients with liver disease.

Acknowledgments The authors wish to thank the many collaborators that contributed to their past and current research studies in people with hepatitis C. Dr Hauser wishes to thank Jirina, Cathy, Katia, Anika and Max Hauser, Alba Pillwein, Dr and Mrs Frido and Laura Kiefhaber, Mr and Mrs Tony and Bev Ostroski and Mr and Mrs Brad and Gretchen Witke for their continued support.

\section{References}

1. Whitehead AJ, Dobscha SK, Morasco BJ, Ruimy S, Bussell C, Hauser P. Pain, substance use disorders and opioid analgesic prescription patterns in veterans with hepatitis C. J Pain Symptom Manag. 2008;36:39-45.

2. Silberbogen AK, Janke EA, Hebenstreit C. A closer look at pain and hepatitis C: Preliminary data from a veteran population. $J$ Rehabil Res Dev. 2007;44:231-244.

3. Rosenblum A, Joseph H, Fong C, Kipnis S, Cleland C, Portenoy RK. Prevalence and characteristics of chronic pain among chemically dependent patients in methadone maintenance and residential treatment facilities. JAMA. 2003;289:2370-2378.

4. El-Serag HB, Kunik M, Richardson P, Rabeneck L. Psychiatric disorders among veterans with hepatitis $\mathrm{C}$ infection. Gastroenterology. 2002;123:476-482.

5. Huckans MS, Blackwell AD, Harms TA, Indest DW, Hauser P. Integrated hepatitis $\mathrm{C}$ virus treatment: addressing comorbid 
substance use disorders and HIV infection. AIDS. 2005; Suppl 3:S106-S115.

6. Loftis JM, Matthews AM, Hauser P. Psychiatric and substance use disorders in individuals with hepatitis C: epidemiology and management. Drugs. 2006;66:155-174.

7. Sylvestre DL, Loftis JM, Hauser P, et al. Co-occurring Hepatitis C, substance use, and psychiatric illness: treatment issues and developing integrated models of care. J Urban Health. 2004;81:719-734.

8. Rogal SS, Winger D, Bielefeldt K, Szigethy E. Pain and opioid use in chronic liver disease. Dig Dis Sci. (Epub ahead of print). doi:10.1007/s10620-013-2638-5.

9. Fireman M, Indest DW, Blackwell A, Whitehead AJ, Hauser P. Addressing tri-morbidity (hepatitis $\mathrm{C}$, psychiatric disorders, and substance use): the importance of routine mental health screening as a component of a comanagement model of care. Clin Infect Dis. 2005; 15:S286-S291.

10. Morasco BJ, Huckans M, Loftis JM, et al. Predictors of pain intensity and pain functioning in patients with the hepatitis $\mathrm{C}$ virus. Gen Hosp Psychiatry. 2010;32:413-418.

11. Campbell LC, Clauw DJ, Keefe FJ. Persistent pain and depression: a biopsychosocial perspective. Biol Psychiatry. 2003;54:399-409.

12. Gatchel RJ, Peng YB, Peters ML, Fuchs PN, Turk DC. The biopsychosocial approach to chronic pain: scientific advances and future directions. Psychol Bull. 2007;133:581-624.

13. Thompson ME, Barkhuizen A. Fibromyalgia, hepatitis $\mathrm{C}$ infection, and the cytokine connection. Curr Pain Headache Rep. 2003;7:342-347.

14. Cacoub P, Poynard T, Ghillani P, et al. Extrahepatic manifestations of chronic hepatitis C. MULTIVIRC Group. Multidepartment Virus C. Arthritis Rheum. 1999;42:2204-2212.
15. Loftis JM, Huckans M, Ruimy S, Hinrichs DJ, Hauser P. Depressive symptoms in patients with chronic hepatitis $\mathrm{C}$ are correlated with elevated plasma levels of interleukin-1beta and tumor necrosis factor-alpha. Neurosci Lett. 2008;430:264-268.

16. Nelligan JA, Loftis JM, Matthews AM, Zucker BL, Linke AM, Hauser P. Depression comorbidity and antidepressant use in veterans with chronic hepatitis C: results from a retrospective chart review. J Clin Psychiatry. 2008;69:810-816.

17. Maes M, Yirmyia R, Noraberg J, et al. The inflammatory \& neurodegenerative (I\&ND) hypothesis of depression: leads for future research and new drug developments in depression. Metab Brain Dis. 2009;24:27-53.

18. Sluzewska A, Rybakowski JK, Laciak M, Mackiewicz A, Sobieska M, Wiktorowicz K. Interleukin-6 serum levels in depressed patients before and after treatment with fluoxetine. Ann NY Acad Sci. 1995;762:474-476.

19. Maes M, Song C, Lin AH, et al. Negative immunoregulatory effects of antidepressants: inhibition of interferon-gamma and stimulation of interleukin-10 secretion. Neuropsychopharmacology. 1999;20:370-379.

20. O'Brien SM, Scully P, Fitzgerald P, Scott LV, Dinan TG. Plasma cytokine profiles in depressed patients who fail to respond to selective serotonin reuptake inhibitor therapy. J Psychiatr Res. 2007;41:326-331.

21. Fishbain DA, Cutler R, Rosomoff HL, Rosomoff RS. Chronic pain-associated depression: antecedent or consequence of chronic pain: A review. Clin J Pain. 1997;13:116-137. 